\title{
Inquiry-Based Learning in Medicine
}

\author{
Thorsten Schäfer
}

At each new encounter with a patient, medical students are challenged to engage in inquiry-based learning, at least implicitly. Currently, great efforts are being made to explicitly integrate inquiry-based learning and research into the curricula in the study of medicine. As such, however, special basic conditions apply to the medical studies.

\subsection{Characteristic Features in the Field of Medicine - Basic Conditions for Inquiry-Based Learning}

The goal in medical training is a physician who is trained scientifically and practically, who is capable of autonomous and independent medical practice, continuing education and constant continued development. Training is intended to provide basic knowledge, abilities and skills in all subjects that are required for comprehensive healthcare for the population. Training to become a physician is carried out on a scientific basis and in a manner that is practice and patient-related [...].

This is what is stated in paragraph 1, subparagraph 1 of the Approbationsordnung für Ärzte or German Medical Licensure Act for Physicians (abbreviated as: ÄApprO; source: Approbationsordnung für Ärzte, 2002, translated), which governs medical studies in Germany down to the smallest detail. It defines the subjects and interdisciplinary areas to be taught, prescribes seminars, courses and internships including total cumulative hours for the part of the studies dealing with fundamental science in Annex 1, specifies what are currently 22 clinical subjects, 14 interdisciplinary areas and 5 block placements as

T. Schäfer, Prof. Dr. med. ( ()

Universität Bochum, Medizinische Fakultät, Studiendekan der Medizinischen Fakultät,

Bochum, Germany

e-mail: Thorsten.Schaefer@rub.de

H. A. Mieg (ed.), Inquiry-Based Learning - Undergraduate Research, https://doi.org/10.1007/978-3-030-14223-0_15 
compulsory study content in paragraph 27 , and defines the type, content and scope of exams within the university and the nationally uniform written state examinations (Staatsexamen) applicable throughout Germany.

The so-called Model Clause (paragraph 41 ÄApprO) in the Medical Licensure Act opens up the possibility of replacing the first state examination, which is focused on the basics, with an equivalent examination, thus enabling greater integration of the curricula and one's areas of focus. Nevertheless, all medical students must pass a written state examination after the 10th semester, and an oral-practical state examination after the 12th semester.

The German institute for medical and pharmaceutical examinations (Institut für medizinische und pharmazeutische Prüfungsfragen or IMPP), which was commissioned to create the national written examinations, publishes what are known as topic catalogs, which list the examination contents as keywords for the basic subjects as well as the clinical and clinical-theoretical subjects. It is not just the relevant textbooks that are geared towards these extensive catalogs and serve to facilitate targeted exam preparation. The courses offered at the university have been subject to the student demand for exam relevance. Courses that are not directly relevant to the examination are, at best, perceived with special interest by few students due to the enormous volume of material and considerable exam pressure in medical studies.

Doubtless specifying the range of subjects, defining the common study content and nationwide examinations for aspiring physicians serves as quality assurance in medical studies; however, it also insulates those studies as compared to most other degree programs that have experienced significant advancements, individualization and reorientation due to the Bologna Process.

Practice orientation and scholarliness are the milestones on which medical studies should focus, according to paragraph 1 of the ÄApprO. As such, the "and" is sometimes also understood as "despite" or "instead of" in the political debate over the provision of care in a country-doctor setting. In the discourse about the orientation of medical studies, however, a shift towards academic qualification is currently being observed.

\subsection{General Experiences with Inquiry-Based Learning in Medical Studies}

Inquiry-based learning does not appear in the framework of medical studies outlined above. As a general rule, medical students are not required to prepare their own academic work. Nonetheless, there is a high percentage of students who often develop interest in medical research during their studies and who pursue a doctoral dissertation in addition to, rather than as part of, their medical studies. At the same time, a very broad range of topics as well as spectrum of quality can be observed. This aspect will be revisited below.

Due to the specifications of the ÄApprO and the IMPP, the curricular internships related to aspects of basic science in physics, chemistry, biology, anatomy, physiology and 
biochemistry are oriented towards promoting an understanding of fundamental relationships and promoting basic skills. Both the independent choice of methods and autonomous analysis and interpretation of the data are lacking, as are the time and remaining resources and ostensibly the exam relevance - to allow experimental approaches with open questions that are to be developed.

At many locations, the proof of performance for the cross-section of "epidemiology, medical biometry and medical information technology" to be provided after the first state examination starting in the 5th semester includes a systematic introduction into scientific work and evidence-based medicine as a compulsory curricular program and allows students to experience the basics of research and the creation and application of new knowledge.

Based on the position paper from the Federal University Assistants' Conference (BAK) from 1970, Huber (2009, p. 9) cited the features of inquiry-based learning specified therein:

- independent selection of the topic,

- independent 'strategy,' in particular with reference to methods, experimental design, research,

- corresponding risk of errors and detours on the one hand, an opportunity for chance discoveries, 'fruitful moments'... on the other,

- working according to the demands of science (e.g. adequate examination of existing knowledge, endurance...),

- self-critical examination of the result with regard to its dependence on hypotheses and methods,

- endeavor to present the achieved result in such a way that its meaning becomes clear and the way in which it was reached is made verifiable.

The fact that "such strongly emphasized independence" represents a high goal, but must first develop over various stages, is relativizing.

Given this understanding, curricular medical studies certainly include a high proportion of inquiry-based learning, albeit learning has hitherto been more implicit: In addition to the theoretical attention, the patient-orientation required in the ÄApprO is provided in the curriculum, in particular through "bedside teaching," block placements, and three 16-week tertiary sessions of full-time clinical-practical work in academic teaching hospitals during the "practical year" at the end of the course of studies. Of a total of 476 hours of teaching at the bedside, half of this must be in the form of a patient demonstration in a group of no more than six, and one patient by a student in a group of no more than three students.

There are direct parallels to the characteristics of inquiry-based learning in the case of the situation at the bedside: Students choose the strategies and methods themselves, build on existing knowledge, and independently formulate hypotheses based on the results of the interview and examination, e.g. in terms of diagnosis and differential diagnoses, investigate and test these hypotheses in a critical and unbiased manner, experience errors and detours, as well as incidental findings and "fruitful moments." Their results must be 
presented in such a manner that the way in which the results were reached is comprehensible and verifiable. With an assumed workload of about 30 credits according to ECTS, this inquiry-based learning at the bedside represents a significant proportion of the curricular teaching in the twelve semesters of medical studies. Not all agents are aware of the significance of this type of instruction in sharpening scientific thinking.

At many locations, for example at the Ludwig-Maximilians-Universität Munich and at the University of Heidelberg, structured doctoral programs have been very consciously set up for medical students in order to provide an optional offering to support independent academic work on the one hand, and in order to improve the quality of medical doctorates in terms of the structural conditions and to sustainably improve the process and the results on the other. These offerings range from support in the application phase of a doctoral project, the mutual obligation between doctoral student and advisor, the guarantee of support and infrastructure, and support through theoretical and practical training to scholarships for doctoral students with particularly ambitious doctoral theses.

Since the ÄApprO was amended in 2002, attempts have been made in some model degree programs, for example the University of Hamburg and the Charité, Berlin, to anchor scientific thinking and activity, and inquiry-based learning, in the curriculum. This is exemplified by the study reforms in the Faculty of Medicine at Ruhr University Bochum, for example.

\subsection{Inquiry-Based Learning Based on the Example of a Model Degree Program and an Integrated Reformed Degree Program}

In 2003, parallel to a reformed standard curriculum, the Faculty of Medicine at Ruhr University Bochum launched a model degree program, which was characterized in particular by a targeted problem, practice and patient orientation. For nine years, 42 students per year were enrolled in this independent, problem-oriented learning degree program. After evaluating this model project, a new "integrated, reformed degree program in medicine" was created, which combined the advantages of the model degree program in medicine with those of a reformed standard curriculum for a large number of students; for all 300 students in the 2013/14 winter semester, and even 330 new students since 2014/15.

\subsubsection{Problem-Based Learning in the Model Degree Program}

The model degree program in medicine dispensed with the systematic transfer of knowledge in lectures. Instead, students were involved in a topic-centered curriculum with concrete patient cases, documented cases in the first four semesters, and then increasingly with real patients. These cases were selected in such a way that general knowledge, clinical theory and clinical knowledge were developed through this involvement. The development followed the classification system of problem-based learning (Schmidt et al. 2011). 
The cases were analyzed and processed in seven steps by groups of seven students, each group being under the guidance of a trained tutor:

1. Clarification of comprehension questions for case presentation

2. Limitation of topics

3. Brainstorming with activation of existing knowledge

4. Forming hypotheses

5. Formulating concrete learning objectives for the group

6. Time for independent learning

7. Presentation and discussion of learning outcomes.

The time provided for independent learning was flanked by courses in the form of seminars and practical tutorial. Using this unbiased approach, students were trained

- to engage with new issues,

- to select the topics themselves,

- to accept the risk of errors and detours, but also to experience "fruitful moments,"

- to use their available knowledge and to research critically,

- to check the results in a self-critical manner and with other group members and to present these results comprehensively.

Problem-based learning was supplemented, inter alia, by a "vertical educational track" anchored in the study regulations on the topics of health economy, scholarliness, methodology and research, in which scientific thinking and work methods were to be presented and built up over the first six semesters, and implemented in students' own tasks and reports in a manner that was exam relevant.

In later semesters, scientific symposia were integrated into the program, in which researchers from the Faculty of Medicine presented their scholarly work and newest findings, and discussed these with students. The problem orientation of this model degree program appears to strengthen the interest in continued autonomous, scholarly work. An initial analysis of the rate at which students obtain doctoral degrees as compared with reformed conventional curriculum suggests this.

This model degree program for 42 new students was planned as a pilot project and was implemented in parallel with a reformed conventional curriculum of approximately 260 new students. On the one hand, this parallelism provided excellent opportunities for researching various educational strategies, especially as the students in the model degree program were chosen by lot from among the applicants; on the other hand, the school posed significant logistical challenges for the faculty. In a two-year planning process that involved multiple departments, a new degree program was therefore developed based on the evaluation results; it has been offered to all new students at the Faculty of Medicine at Ruhr University Bochum as an "integrated, reformed degree program in medicine" since the 2013/14 winter semester. 


\subsubsection{Inquiry-Based Learning and Learning to Research in the Reformed Degree Program}

Inquiry-based learning is now anchored in the curriculum in three stages:

The basic, systematic scientific education in the first four semesters, or the first stage, is accompanied by problem-based learning, in which concrete, topic-oriented patient cases are processed based on the aforementioned seven steps: Hypothesis formation based on knowledge in a scientifically founded excursus with the other members of the team, research in the event that there are open questions as well as the presentation, critical questioning and fact-based defense of the learning outcomes are the main features of researchoriented, unbiased and self-determined learning. The tutors, all of the lecturers at the Faculty of Medicine, thereby have the task of "allowing" the group process "to play out," and they should introduce as little as possible in terms of content.

In stage two, which is obligatory for all students, the foundation of scientific thinking and working are systematically processed. This occurs in compulsory courses as lectures and practical tutorials during the fifth semester within the context of the proof of performance for "epidemiology, medical biometry and medical information technology." At the end of the semester there is a written exam on theoretical knowledge. Learning objectives during this stage include not just getting to know and assessing research findings, but also internalizing the research process itself, from the development of a precise research question about the suitable choice of method, the analysis, and the presentation and critical discussion of the results to classifying these in the current state of research.

In stage three, students must select a main area in which they wish to deepen their knowledge of previously learned theory in small groups and in scholarly discourse. There are three topics to choose from in sixth-semester seminars:

1. Basic biomedical research

2. Clinical research

3. Evidence-based medicine

Organized beneath these three main themes are various small group courses focused on ongoing research projects, which provide the participants with immediate, practiceoriented insight into the research process and room for their own research-based work.

\subsection{Outlook for Inquiry-Based Learning in Medicine - What Needs to Be Done?}

The call for stronger practice orientation in medical training has grown in recent years as part of the concerns surrounding providing medical care to the population. At the same time, however, there is a growing conviction that the doctors we are training today not only need to have internalized the present "state of the art" and must act with great knowledge, 
sophisticated practical skills and appropriately for the profession. As medical advances continue to increase, today's graduates must also be able to understand the advances of tomorrow (and beyond) that are relevant to them, to analyze the relevancy of those advances for their own patients and utilize them for those patients' benefit, and must be as prepared and able as possible to contribute to these advances themselves.

\subsubsection{Recommendations of the German Science Council (Wissenschaftsrat) for the Further Development of Medical Studies}

In 2012, the German Science Council started the work program "Stand und Perspektiven der humanmedizinischen Modellstudiengänge" ("Status of and perspectives on model degree programs in human medicine"). In this program, ongoing degree programs were examined with respect to the question of which reform elements have been successful, and what conclusions could be drawn for the advancement of medical studies in Germany.

Concerning this, in 2014 the German Science Council presented its "Empfehlungen zur Weiterentwicklung des Medizinstudiums in Deutschland auf Grundlage einer Bestandsaufnahme der humanmedizinischen Modellstudiengäng" ("Recommendations for the further development of medical studies in Germany on the basis of a survey of model degree programs in human medicine") (Wissenschaftsrat 2014). The recommendations are summarized in five principles. These deal with (1) competence orientation, (2) integrated patient-oriented curricula, (3) scientific competencies, (4) interprofessional education and (5) focusing the curricular content. With regard to item 3, which is relevant here, the German Science Council stated the following in its abstract (p. 7, translated):

Scientific competencies: Physicians must be able to examine their own actions in more complex care situations with regard to basing them in evidence and against the background of new medical findings, in order to arrive at a decision that relates to the individual patient. Thus scientific thinking and activity forms the basis for the adequate, patient-oriented selection of diagnostic and therapeutic measures. The compulsory acquisition of scientific competencies in the course of studies is thus a necessary prerequisite for responsible medical practice.

In concrete terms, the German Science Council recommends the introduction of a longitudinal educational track, the courses or modules of which build on one another, extending over multiple semesters with the aim "that knowledge, skills and attitudes toward scientific thinking and activity when working through problems in practice, as well as the process of scientific knowledge generation and assurance can be practiced by students themselves." The format of problem-based teaching has proven itself in its practical application.

What is new for medical studies - but common for nearly all other degree programs - is that conducting one's own research is required, in the course of which scientific competency is to be proven, whereby this research is to become an elementary component of the 
curriculum. It is stated that the German Science Council is aware of the fact that this will necessitate a cultural change in medicine. The arguments are easy to understand, but at the same time, practical questions are raised regarding the current basic conditions outlined above, which likewise must be reformed in the sense of the advancement of medical studies in Germany. This also includes providing the necessary resources, because the abovementioned requirements suggest a considerable additional support effort.

\subsubsection{New: A National Competency-Based Catalog of Learning Objectives for Medicine (NKLM)}

A very concrete, promising approach to the establishment of inquiry-based learning in medicine can be found in the development of the national competency-based catalog of learning objectives for medicine (NKLM) and for dentistry (NKLZ) (Hahn and Fischer 2009). The Gesellschaft für Medizinische Ausbildung (Society for Medical Education) and the Medizinische Fakultätentag (German Medical Faculty Association), in coordination with the member societies of the Arbeitsgemeinschaft der Wissenschaftlichen Medizinischen Fachgesellschaften (Working Group of Scientific Medical Societies), are currently working on a multidimensional catalog of competencies and operationalized learning objectives which should be acquired by the end of twelve semesters of medical studies. In the process, the physician should not be seen as just a "medical expert" with a wealth of medical knowledge, but rather it is stressed that additional roles are integrated into this expertise. Based on the CanMEDS framework of the Royal College in Canada (actually developed for medical continuing education), in the NKLM and NKLZ, physicians' roles as scholars, communicators, team members, health advisors and advocates, responsible parties, managers and professionals have been underpinned by corresponding competencies.

The subject of "inquiry-based learning in medicine" is expressed here in particular in the physician's role as scholar. Here, the NKLM defines four core competencies, the first of which is that graduates are lifelong learners, who improve and maintain their professional activities by means of constant continued education. Critical reflection on one's own level of knowledge, uncovering knowledge gaps, defining one's own learning needs, effective implementation of suitable learning strategies and adequate documentation and implementation of the learning outcomes for the benefit of the patients are specific goals in this category.

Secondly, as defined by the NKLM, physicians should be able to critically evaluate scientific information and the sources of that information, and should be able to apply it in a suitable manner to their own actions. Here, the NKLM integrates the principles of evidence-based medicine, which are both credo and challenge to science-based, individualized medicine. 
Thirdly, scholarliness also includes teaching competencies for various target groups. Here, the education and instruction of patients and relatives, and the training of medical students and students in other health professions, is explicitly specified.

Fourthly, as innovators, aspiring physicians should contribute to the formation, dissemination, application, and translation of new insights and practices. In order to develop and practice these competencies, physicians are recommended to do their own practical work, going beyond the understanding of the philosophy of science and the knowledge of ethical and legal principles of research. This includes research work including the following stages: the derivation of a research question and testable hypothesis; systematically obtaining information on the current state of the research; and planning and carrying out a research project, including documentation and disseminating the research findings. Thus the research activities correspond to the recommendations of the German Science Council for strengthening scientific competencies within the context of the advancement of medical studies in Germany.

That such an integration of inquiry-based learning and scientific work in the medical studies curriculum is possible is shown by the advancement of medical studies abroad. Within the context of the Bologna reform, for example, medical degree programs in the Netherlands and Switzerland were converted into graduated, modularized degree programs, in which an independent scientific thesis within the context of the bachelor's and/ or master's degree program is obligatory.

\subsection{Conclusion}

The variety of new projects on inquiry-based learning and on scholarly thinking and working at medical schools, the recommendations of the German Science Council for the advancement of medical studies in Germany and the agreement on scientific competencies in the national competency-based catalog of learning objectives for medicine and dentistry are conspicuous signs of a "change in culture" in medicine towards integrating independent, scholarly work in the medical curriculum (Fischer and Fabry 2014). It is to be hoped that this change in culture will be constructively and rapidly accompanied by a change in the basic conditions and will inspire scholarliness and a practice orientation in the course of studies.

\section{References}

Approbationsordnung für Ärzte vom 27. Juni 2002 (BGB1. I S. 2405), die zuletzt durch Artikel 2 der Verordnung vom 2. August 2013 (BGBl. I S. 3005) geändert worden ist Bundesgesetzblatt I 2405, Bundesministerium für Gesundheit 2002.

Fischer, M. R./Fabry, G. (2014). Thinking and acting scientifically: Indispensable basis of medical education. GMS Zeitschrift für medizinische Ausbildung, 31(2), Doc24. doi:https://doi. org/10.3205/zma000916. 
Hahn, E. G./Fischer, M. R. (2009). Nationaler Kompetenzbasierter Lernzielkatalog Medizin (NKLM) für Deutschland: Zusammenarbeit der Gesellschaft für Medizinische Ausbildung (GMA) und des Medizinischen Fakultätentages (MFT). GMS Zeitschrift für medizinische Ausbildung, 26(3), Doc35. Retrieved 05 May 2015 from http://www.egms.de/en/journals/zma/2009-26/zma000627. shtml

Huber, L. (2009). Warum Forschendes Lernen nötig und möglich ist. In L. Huber/J. Hellmer/ F. Schneider (Hrsg.), Forschendes Lernen im Studium. Aktuelle Konzepte und Erfahrungen. (S. 9-35). Bielefeld: UniversitätsVerlagWebler.

Institut für medizinische und pharmazeutische Prüfungsfragen (2015). Gegenstandskataloge für den schriftlichen Teil des Ersten Abschnitts der Ärztlichen Prüfung (IMPP-GK1) und Gegenstandskataloge für den schriftlichen Teil des Zweiten Abschnitts der Ärztlichen Prüfung (IMPP-GK2). Retrieved 05 May 2015 from https://www.impp.de/internet/de/medizin/articles/ gegenstandskataloge.html

Royal College of Physicians and Surgeons of Canada (o.J.). The CanMEDS Framework. Retrieved 05 May 2015 from http://www.royalcollege.ca/portal/page/portal/rc/canmeds/framework

Schmidt, H. G./Rotgans, J. I./Yew, E.H.J. (2011). The process of problem-based learning: what works and why. Medical education, 45(8), 792-806. doi:https://doi.org/10.1111/ j.1365-2923.2011.04035.x.

Wissenschaftsrat (2014). Empfehlungen zur Weiterentwicklung des Medizinstudiums in Deutschland auf Grundlage einer Bestandsaufnahme der humanmedizinischen Modellstudiengänge. Köln: Wissenschaftsrat.

Open Access This chapter is licensed under the terms of the Creative Commons AttributionNonCommercial-NoDerivatives 4.0 International License (http://creativecommons.org/licenses/bync-nd/4.0/), which permits any noncommercial use, sharing, distribution and reproduction in any medium or format, as long as you give appropriate credit to the original author(s) and the source, provide a link to the Creative Commons licence and indicate if you modified the licensed material. You do not have permission under this license to share adapted material derived from this chapter or parts of it.

The images or other third party material in this chapter are included in the chapter's Creative Commons licence, unless indicated otherwise in a credit line to the material. If material is not included in the chapter's Creative Commons licence and your intended use is not permitted by statutory regulation or exceeds the permitted use, you will need to obtain permission directly from the copyright holder. 